\title{
Association between rs7517847 and rs2201841 polymorphisms in IL-23 receptor gene and risk of ankylosing spondylitis: A meta-analysis
}

Bin Xu, Jian-xiong Ma, Xin-long Ma, Hao-bo Jia, Rui Feng, Li-yan Xu

To comprehensively evaluate the association between rs7517847 and rs2201841 polymorphisms in the Interleukin-23 (IL-23) receptor gene and ankylosing spondylitis (AS), a meta-analysis was performed. The Pubmed, Embase, MEDLINE, Cochrane, China National Knowledge Infrastructure (CNKI), VIP, Wanfang and China Biology Medicine disc (CBMdisc) databases were searched to identify eligible studies on rs7517847 and rs2201841 polymorphisms in the IL-23 receptor gene and AS that were published through September 2014. Data of interest were extracted from each study, and the meta-analysis was performed using STATA 12.0. Four studies were eligible for the meta-analysis and included a total patient population of 2,465 . With regards to rs 7517847 , the current study showed that the genotype $G G$ and allele $G$ might play a protective role during $A S(O R=0.76,95 \%$ $\mathrm{Cl}=0.59-0.99 ; \mathrm{OR}=0.88,95 \% \mathrm{Cl}=0.78-0.99$ for homozygote and allelic models, respectively). However, according to the meta-analysis, there was no statistical association between the genotype or allele of rs2201841 and an individual's susceptibility to AS in all genetic models. In conclusion, it was the IL-23 rs7517847 polymorphism rather than the rs2201841 polymorphism that had a statistical association with AS. Nevertheless, more evidence is needed to confirm this result. Consequently, it is necessary to carry out more high-quality studies to confirm the associations between these two single nucleotide polymorphisms and AS. 


\section{Bin Xu ${ }^{1,2}$, Jian-xiong Ma ${ }^{1}$, Xin-long Ma ${ }^{1}$, Hao-bo Jia ${ }^{1}$, Rui Feng ${ }^{1}$, Li-yan Xu' \\ ${ }^{1}$ Biomechanics Laboratory of Orthopaedic Institute of Tianjin Hospital, Tianjin, China \\ ${ }^{2}$ Tianjin Medical University, Tianjin, China}

Corresponding author: Xin-long $\mathrm{Ma}$

E-mail: pubstory@163.com

Fax: 022-60362637

\section{Introduction}

Ankylosing spondylitis (AS) is an autoimmune disease that features chronic inflammation and ossification of the sacroiliac articulation and enthesis of the tendon. Approximately $0.2 \%$ of the general population suffers from AS, and its incidence is $0.1-1.4 \%, 0.2-0.54 \%, 0.86 \%$, and $2 \%$ in European, Chinese, Caucasian, and HLA-B27-positive populations, respectively ${ }^{[1-4]}$. Moreover, when one twin suffers from AS, the probability that the other is also an AS patient is greater than $90 \%[5]$. An obvious gender tendency has been found in AS, i.e., men are more susceptible to the disease compared with women $^{[4]}$.

The etiology and mechanisms behind AS remain unclear. Scientists have thought that susceptibility to AS is strongly affected by heredity. Although HLA-B27 is a confirmed genetic risk factor, not all AS cases can be explained by genetic association, which implies that other factors are also important ${ }^{[6]}$. Wang et al. found that there was a significantly higher mRNA expression of the interleukin-23 receptor (IL-23R) gene in peripheral blood monocytes of AS patients compared with those of normal controls ${ }^{[7]}$. When IL-23 was overexpressed, CD4- and CD8- T cells were affected, ${ }^{[8]}$ which led to peripheral enthesitis and the formation of new bone ${ }^{[9]}$. Another study showed that the IL-17/IL-23 axis could be a potential target for AS treatment ${ }^{[10]}$. In accordance with a genome-wide association study (GWAS), it was also shown that there was an association between IL-23R and AS in Caucasian patients ${ }^{[11]}$.

Thus far, several meta-analyses have investigated the relationship between several single nucleotide polymorphisms (SNPs) and AS; they have found that several IL-23R SNPs were associated with AS in Caucasian populations but that others were unrelated to AS in Asian patients ${ }^{[12-15]}$. No meta-analysis has yet examined the relationship between SNPs rs7517847 and rs2201841 and AS. The association between these two SNPs and AS was borderline significant only ${ }^{[16,17]}$. These negative results may have been caused by a small included population. The current study uses meta-analysis to determine the association between rs7517847 and rs2201841 in IL-23R SNPs and AS for the first time. This study provides more comprehensive evidence for rheumatologists considering the association between IL23R SNPs and AS.

\section{Materials and Methods}

Search Strategy and Selection Criteria

A comprehensive search of databases such as Pubmed, Embase, Medline, Cochrane, China National Knowledge Infrastructure (CNKI), VIP, Wanfang and China Biology Medicine disc (CBMdisc) databases was conducted. Searches included literature dated from database origin to September 2014, and the following key words were used: "IL-23" OR "interleukin-23", "Ankylosing Spondylitis" OR 
"AS", and "polymorphism" OR "polymorphisms". In the CNKI, VIP, Wanfang and CBMdisc databases we searched for corresponding words in Chinese characters. The full search strategy for the Embase database is presented in Table 1. No language restrictions were used. A manual search for references beyond those in the above-mentioned databases was also implemented. For studies that did not describe genetic distribution data in detail, email correspondence with the main authors was used to complete the data. Titles and abstracts were independently screened by two authors to identify potentially related studies. Full-text versions of the identified studies were reviewed to select those that met the eligibility criteria. The identified studies were subjected to a final confirmation before inclusion in the meta-analysis.

\section{Inclusion Criteria:}

Investigations that met the eligibility criteria were included in the analysis, and any disagreements were resolved by discussion between the authors (Xu B and Ma JX). In cases where a consensus could not be reached, a third author was involved to make a final decision. Studies meeting the following criteria were included in the meta-analysis: (1) investigation evaluating the association between IL-23R rs7517847 or rs2201841 polymorphisms and AS susceptibility; (2) a case-control study or GWAS; (3) sufficiently available public data that could be extracted for further analysis, such as genotype distribution, odds ratio (OR) and 95\% confidence interval (95\% CI); and (4) a SNP distribution according to Hardy-Weinberg equilibrium (HWE) was included, which means high quality in design and conduct of genetic association studies. In cases where two studies examined the same or overlapping populations, the study with a larger sample size was included in the analysis.

Studies for which contact with the main author could not be reached to supply information about relevant data were excluded.

\section{Data Extraction and Quality Assessment}

The relevant characteristics of the included investigations were identified and recorded by two authors, including the first author of the study, publication year, country, ethnicity of subjects, relevant SNPs, patient demographics, test method used for genotype, whether the genotype distribution was in accordance with Hardy-Weinberg Equilibrium (HWE) and source of the samples tested. To acquire precise results for the current study, email contact with the main author of the investigation was performed if the included study did not contain public data. Quality assessment of studies included in the meta-analysis was conducted by two authors using the Newcastle-Ottawa Scale (NOS) ${ }^{[18]}$. Scores were given for subject selection (i.e., adequateness of the case definition, representativeness of the cases, selection of controls, and definition of controls) and the comparability of the groups (i.e., comparability of cases and controls on the basis of the design or analysis) as well as measurement of exposure (i.e., ascertainment of exposure, same method of ascertainment for cases and controls, and non-response rate). NOS scores ranged from 0 to 9 . Studies with a NOS score $\geq 6$ were considered to be high quality. Higgins $I^{2}$ was used to evaluate the heterogeneity of the investigations. Subgroup analysis by ethnicity was executed if the number of investigations in each ethnic group was two or more. Gender subgroup analysis could not be done because there was no relevant data. Sensitivity analysis was carried out by evaluating the overall results of meta-analysis when each study was removed to detect the stability of trials included.

Statistical Analysis 
The strength of the association between IL-23R rs7517847 and rs2201841 polymorphisms and AS susceptibility was evaluated using the Odds Ratio (OR). At the same time, precision was measured by 95\% CI. A random-effects model was used in the current study. With respect to rs7517847 and rs2201841, the homozygote model, heterozygote model, recessive model, dominant model and allelic model were used to estimate susceptibility to AS in current study. Statistical analysis of the extracted data was carried out using STATA 12.0.

\section{Results}

\section{Search Results}

As a result of the search strategy presented in Figure 1, 215 English-language studies and 208 Chinese-language studies were obtained. In addition, one study was added by searching through references $^{[19]}$. Among the search results, 420 trials were excluded for the following reasons: (1) 116 were duplicate articles; (2) 281 did not discuss the association between IL-23R SNPs and AS in the title and abstract; (3) 20 did not discuss the association between rs7517847 and rs2201841 and AS in the full-text; (4) two contained genotype distribution data that were not publicly available, and email contact with the main authors could not be achieved; and (5) two studies assessed overlapping populations $[16,20]$. For these studies, those that included a larger sample population were included in the analysis, as conducted by Safrany et al ${ }^{[16]}$. Finally, four trials ${ }^{[16,17,19,21]}$ on rs 7517847 and two trials ${ }^{[16,19]}$ on rs2201841 were included in the current study. The included studies had case-control designs with 1,006 patients in the AS group and 1,190 people in the control group for rs 7517847 . For rs2201841, 322 patients were included in the AS group, and 255 people were included in the control group in the current study. The basic characteristics of included studies were listed in Table 2. Patients who were diagnosed with AS according to the New York modified criteria ${ }^{[22]}$ and healthy people from Chinese, Hungary and Spanish populations were included in the current study. All trials included in this meta-analysis were replication studies according to HWE. The NOS score of studies included in the meta-analysis ranged from 6 to 7, as presented in Table 3. Moreover, genotype distribution of both SNPs in the AS and control groups is presented in Table 4.

\section{Overall results of the meta-analysis}

ORs, $\mathrm{P}$ value, 95\% CI and heterogeneity evaluation of meta-analysis for rs 7517847 and rs 2201841 are presented in Table 5 and Figure 2 and in Table 6 and Figure 3, respectively. The random effect model was applied to the studies. A comprehensive analysis of relevant SNPs was performed. Based on the meta-analysis results, there was a statistically significant association between the IL-23R rs7517847 polymorphism in the over-all population and AS susceptibility under two genetic models (homozygote model, i.e., GG vs. TT, were $\mathrm{OR}=0.76,95 \% \mathrm{CI}=0.59-0.99$ and $\mathrm{P}=0.038$ and the allelic model, $\mathrm{G}$ vs. T, i.e., where $\mathrm{OR}=0.88,95 \% \mathrm{CI}=0.78-0.99$ and $\mathrm{P}=0.032$ ). The heterogeneity among studies under the two genetic models was $\mathrm{I}^{2}=0, \mathrm{P}_{\mathrm{H}}=0.547$ and $\mathrm{I}^{2}=0, \mathrm{P}_{\mathrm{H}}=0.838$, respectively, which indicated that there was no statistically significant heterogeneity among the trials for rs 7517847 . The analytical results presented in Table 6 implied that there was no statistically significant association between the IL-23R rs2201841 polymorphism and AS susceptibility under all of the genetic models tested.

\section{Results of subgroup analysis by ethnicity}

A subgroup analysis by ethnicity (Asians and Caucasians) was conducted for rs7517847, and 
contained two trials (one with 435 and 455 people in the case and controls groups and a second with 571 and 735 people in the case and control group, respectively). The random effect model was applied to each genetic model in both the Asian and Caucasian populations

In accordance with the results of the subgroup analysis, there was no statistically significant association between the IL-23R rs7517847 polymorphism and AS in the Asian or Caucasian populations, as presented in Table 7.

\section{Sensitivity analysis}

A sensitivity analysis of trials investigating rs 7517847 was carried out, aiming to evaluate the stability of the current study. After removing the study conducted by Safrany et al. ${ }^{[16]}$, we found that the $\mathrm{OR}$ for $\mathrm{GG}$ vs TT changed from $\mathrm{OR}=0.76(95 \% \mathrm{CI}=0.59-0.99, \mathrm{P}=0.038)$ to $\mathrm{OR}=0.80(95 \%$ $\mathrm{CI}=0.60-1.06$ and $\mathrm{P}=0.120)$ and the $\mathrm{OR}$ for $\mathrm{G}$ vs $\mathrm{T}$ changed from $\mathrm{OR}=0.88(95 \% \mathrm{CI}=0.78-0.99$, $\mathrm{P}=0.032)$ to $\mathrm{OR}=0.88(95 \% \mathrm{CI}=0.77-1.01$ and $\mathrm{P}=0.078)$. After removing the study conducted by Dong et al. ${ }^{[17]}$, we found that the $\mathrm{OR}$ for $\mathrm{GG}$ vs TT changed from $\mathrm{OR}=0.76(95 \% \mathrm{CI}=0.59-0.99, \mathrm{P}=0.038)$ to $\mathrm{OR}=0.82(95 \% \mathrm{CI}=0.60-1.11$ and $\mathrm{P}=0.203)$ and the $\mathrm{OR}$ for $\mathrm{G}$ vs $\mathrm{T}$ changed from $\mathrm{OR}=0.88(95 \% \mathrm{CI}=$ $0.78-0.99, \mathrm{P}=0.032)$ to $\mathrm{OR}=0.90(95 \% \mathrm{CI}=0.78-1.04$ and $\mathrm{P}=0.145)$. Excluding the other two studies, we found that the results of studies had not changed.

\section{Discussion}

As a member of the erythropoietin receptor superfamily, IL-23R influences the differentiation of CD4 T lymphocytes to IL-17-producing Th17 lymphocytes. During inflammation, IL-17 plays a destructive role, such as articulation of the cerebral, heart, lung and intestinal tissues. Several recent studies have indicated an association between IL-23R polymorphism and some autoimmune diseases, such as inflammatory bowel disease, multiple sclerosis and psoriasis ${ }^{[23-25]}$. Overall, four relevant metaanalyses about IL-23R and AS have been conducted and provided statistical evidence for the association between IL-23R polymorphisms and AS susceptibility ${ }^{[12-15]}$. The results of two metaanalyses of large populations indicated that associations between 7 SNPs and 6 SNPs of IL-23R and AS existed in Britain and 7 other countries, respectively ${ }^{[12,14]}$.

However, no associations existed for some SNPs in a Portuguese population ${ }^{[15]}$ or five SNPs in an Asian population ${ }^{[13]}$, in studies where fewer than 1,000 individuals were examined. Additionally, a number of other investigations have assessed the association between IL-23R rs7517847 and rs2201841 polymorphisms and AS susceptibility. However, the outcomes were not conclusive. To obtain a statistically credible result, four studies were included in this meta-analysis. In total, four trials (1,006 AS patients and 1,190 people in the control group) and two trials (322 AS patients and 255 people in the control group) were included for rs 7517847 and rs2201841, respectively.

Although no statistically significant association between IL-23R rs7517847 and AS were identified in each of the included studies, the results of the current study showed that there was a statistically significant association between rs7517847 and AS in the overall population in the homozygote model and the allelic model after the four studies were pooled. We speculated that negative results shown in each study were due to the small sample sizes. Additionally, no evidence was obtained to prove the association between rs 7517847 and ethnicity in the subgroup analysis. After excluding either the study conducted by Safrany et al. or that Dong et al., which were conducted in Hungary or China, respectively, we found that the $\mathrm{P}$ value of the remaining studies changed to be not significant. However, 
the odds ratio of the studies were actually fairly stable. (Safrany et al.: as for GG vs TT, OR=0.80, 95\% $\mathrm{CI}=0.60-1.06$ and $\mathrm{P}=0.120$; with respect to $\mathrm{G}$ vs $\mathrm{T}, \mathrm{OR}=0.88,95 \% \mathrm{CI}=0.77-1.01$ and $\mathrm{P}=0.078$ Dong et al.: $\mathrm{GG}$ vs TT: $\mathrm{OR}=0.82,95 \% \mathrm{CI}=0.60-1.11$ and $\mathrm{P}=0.203 ; \mathrm{G}$ vs $\mathrm{T}: \mathrm{OR}=0.90,95 \% \mathrm{CI}=0.78-1.04$ and $\mathrm{P}=0.145)$. The sensitivity analysis was affected by removing the result of either Safrany et al. or Dong et al., possibly because the remaining sample size after removal of these studies was not sufficiently large. Thus, removing a study could greatly reduce the sample size and lead to changes in the sensitivity analysis. Additionally, no significant association between rs2201841 and AS susceptibility was found in any of the genetic models tested in the current study. The results obtained in this study suggest that the rs71517847 polymorphism might be a protective factor for AS in the overall population but that the rs2201841 polymorphism might not be associated with AS susceptibility. The author $(\mathrm{Xu} \mathrm{B})$ speculated that the negative results regarding rs2201841 could have been due to small sample size.

The current study has some limitations as follows. (1) The population included in this study was relatively small. Hence, type II error might exist and the credibility of the current study's results may be weak. (2) A subgroup analysis of rs2201841 by ethnicity could not be conducted because the eligible studies in each ethnicity that were included in this study only involved one trial such that the association between IL-23R rs2201841 and AS by ethnicity was unknown. (3) There was only one country from Asia and two countries from Europe involved in the current study, which implies that the results of the current study are not representative. (4) Unfortunately, there is a lack of information about related studies for the black population. Therefore, the results of the current study are not comprehensive. (5) We were unable to include studies that have not been published, which might affect the publication bias.

\section{Conclusion}

In conclusion, the present meta-analysis showed that the IL-23R rs7517847 polymorphism may play a protective role in AS. However, no association between the rs2201841 polymorphism and AS susceptibility was found. Moreover, to obtain a credible and comprehensive conclusion to enable rheumatologists and researchers in related fields to comprehend the association between rs 7517847 and rs2201841 and AS susceptibility, it is still essential to implement further investigations with a large number of samples from more countries to assess the associations between these two SNPs and AS.

\section{Reference}

[1] Braun J, Sieper J. Ankylosing spondylitis[J]. Lancet,2007(369):1379-1390.

[2] Zeng Q, Chen R, Darmawan J, et al. Rheumatic Diseases in China[J]. Arthritis Research \& Therapy,2008,10(1):R17.

[3] Braun J, Bollow M, Remlinger G, et al. Prevalence of spondylarthropathies in HLA-B27 positive and negative blood donors[J]. Arthritis Rheum,1998,1(41):58-67.

[4] Goldman L, Schafer A I. Goldman's Cecil Medicine[M]. 24 ed. USA:2012.1692-1694.

[5] Brown M, Gail K L, Macgregor A, et al. Susceptibility to ankylosing spondylitis in twins: the role of genes, HLA and the environment[J]. Arthritis Rheum,1997,10(40):1823-1828.

[6] Vegvari A, Szabó Z, Szántó S, et al. The genetic background of ankylosing spondylitis[J]. Joint Bone Spine,2009,76(6):623-628. 
206 [7] Wang X, Huang J, Lin Z, et al. Single-nucleotide polymorphisms and expression of IL23R in Chinese ankylosing

207

208

209

210

211

212

213

214

215

216

217

218

219

220

221

222

223

224

225

226

227

228

229

230

231

232

233

234

235

236

237

238

239

240

241

242

243

244

245

246 spondylitis patients[J]. Rheumatology International,2010,30(7):955-959.

[8] Sherlock J P, Joyce-Shaikh B, Turner S P, et al. IL-23 induces spondyloarthropathy by acting on ROR- $\gamma \mathrm{t}+$ CD3+CD4-CD8- entheseal resident T cells[J]. Nature Medicine,2012,18(7):1069-1076.

[9] Miceli-Richard C. Significant linkage to spondyloarthropathy on 9q31-34[J]. Human Molecular Genetics,2004,13(15):1641-1648.

[10] Di Meglio P, Villanova F, Napolitano L, et al. The IL23R A/Gln381 Allele Promotes IL-23 Unresponsiveness in Human Memory T-Helper 17 Cells and Impairs Th17 Responses in Psoriasis Patients[J]. Journal of Investigative Dermatology,2013,133(10):2381-2389.

[11] Burton P R, Clayton D G, Cardon L R, et al. Association scan of 14,500 nonsynonymous SNPs in four diseases identifies autoimmunity variants[J]. Nature Genetics,2007,39(11):1329-1337.

[12] Karaderi T, Harvey D, Farrar C, et al. Association between the interleukin 23 receptor and ankylosing spondylitis is confirmed by a new UK case-control study and meta-analysis of published series[J]. Rheumatology,2009,48(4):386-389.

[13] Chen C, Zhang X, Li J, et al. Associations of IL-23R polymorphisms with ankylosing spondylitis in East Asian population: a new case-control study and a meta-analysis[J]. International Journal of Immunogenetics,2012,39(2):126-130.

[14] Duan Z, Pan F, Zeng Z, et al. Interleukin-23 receptor genetic polymorphisms and ankylosing spondylitis susceptibility: a meta-analysis[J]. Rheumatology International,2012,32(5):1209-1214.

[15] Pimentel-Santos F M, Ligeiro D, Matos M, et al. Association of IL23R and ERAP1 genes with ankylosing spondylitis in a Portuguese population[J]. Clin Exp Rheumatol,2009,27(5):800-806.

[16] Sáfrány E, Pazár B, Csöngei V, et al. Variants of the IL23R Gene are Associated with Ankylosing Spondylitis but not with Sjögren Syndrome in Hungarian Population Samples[J]. Scandinavian Journal of Immunology,2009,70(1):68-74.

[17] Dong H, Li Q, Zhang Y, et al. IL23R Gene Confers Susceptibility to Ankylosing Spondylitis Concomitant with Uveitis in a Han Chinese Population[J]. PLoS ONE,2013,8(6):e67505.

[18] Cota G F, de Sousa M R, Fereguetti T O, et al. Efficacy of Anti-Leishmania Therapy in Visceral Leishmaniasis among HIV Infected Patients: A Systematic Review with Indirect Comparison[J]. PLoS Neglected Tropical Diseases, 2013,7(5):e2195.

[19] Zhu X, Yang Z, Gao S. The Candidate Gene-Association Study of Ankylosing Spondylitis in Chinese North Population the Study of Pathogenesis of DA2B [in Chinese][J]. ZhongGuo XieHe YiKeDaXue,2009.

[20] Szabo M, Safrany E, Pazar B, et al. Marked diversity of IL23R gene haplotype variants in rheumatoid arthritis comparing with Crohn's disease and ankylosing spondylitis[J]. Molecular Biology Reports,2013,40(1):359-363.

[21] Rueda B, Orozco G, Raya E, et al. The IL23R Arg381Gln non-synonymous polymorphism confers susceptibility to ankylosing spondylitis[J]. Annals of the Rheumatic Diseases,2008,67(10):1451-1454.

[22] Goei The H S, Steven M M, VAN DER Linden S M, et al. EVALUATION OF DIAGNOSTIC CRITERIA FOR ANKYLOSING SPONDYLITIS: A COMPARISON OF THE ROME, NEW YORK AND MODIFIED NEW YORK CRITERIA IN PATIENTS WITH A POSITIVE CLINICAL HISTORY SCREENING TEST FOR ANKYLOSING SPONDYLITIS[J]. Rheumatology,1985,24(3):242-249.

[23] Duerr R H, Taylor K D, Brant S R, et al. A Genome-Wide Association Study Identifies IL23R as an Inflammatory Bowel Disease Gene[J]. Science,2006,314(5804):1461-1463.

[24] Cargill M, Schrodi S J, Chang M, et al. A large-scale genetic association study confirms IL12B and leads to the identification of IL23R as psoriasis-risk genes[J]. Am J Hum Genet,2007,80(2):273-290.

[25] Nunez C, Dema B, Cénit M, et al. IL23R: a susceptibility locus for celiac disease and multiple sclerosis?[J]. Genes and 
247 Immunity,2008,9(4):289-293.

248 


\section{PRISMA flowchart of number of studies}

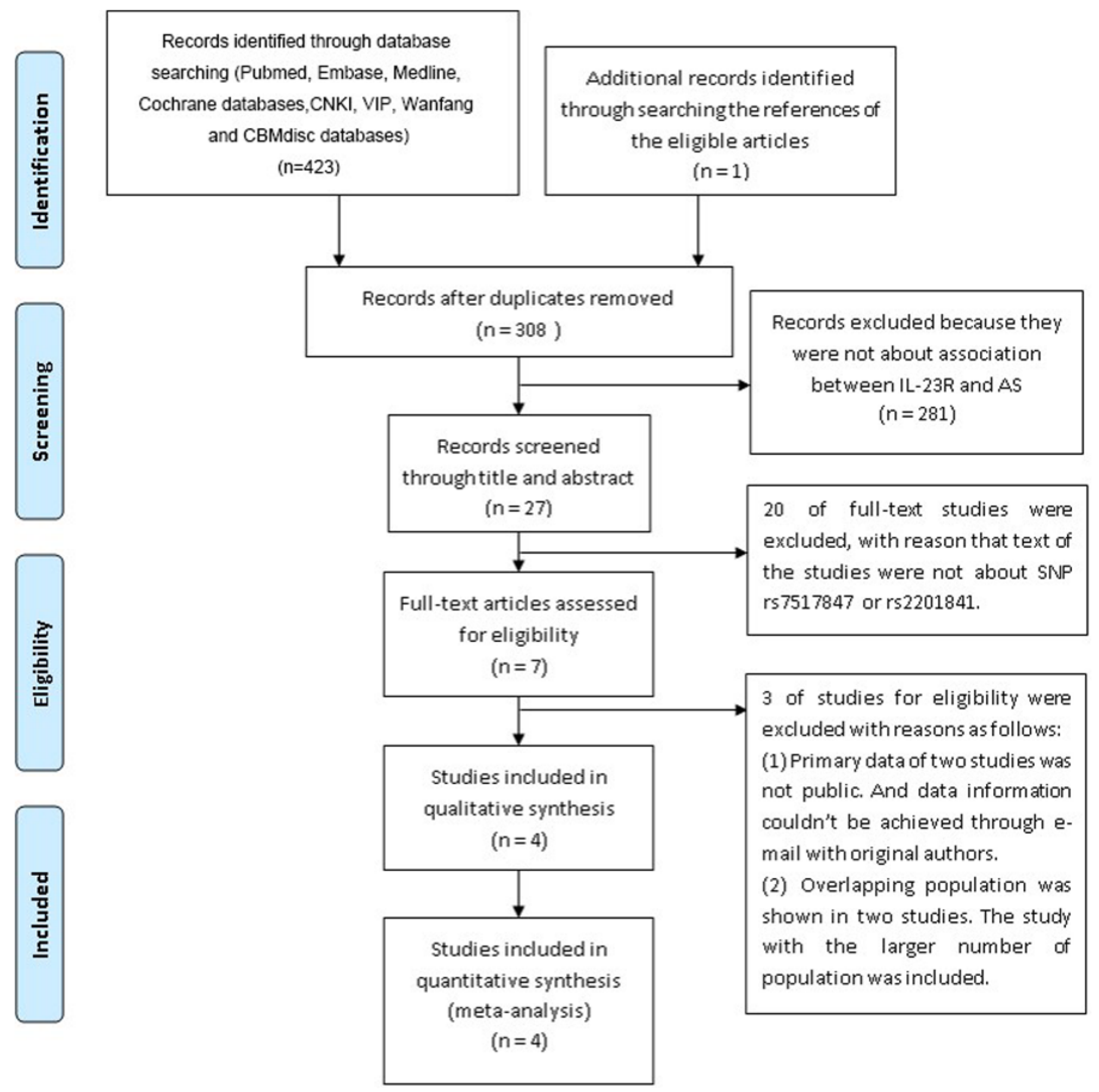


2

Forest plot of genetic association studies of rs7517847

A. genotype GG vs TT; B. allele G vs allele T

The horizontal axis: axis of Odds Ratio (OR)

The dotted line: mean value of overall OR 


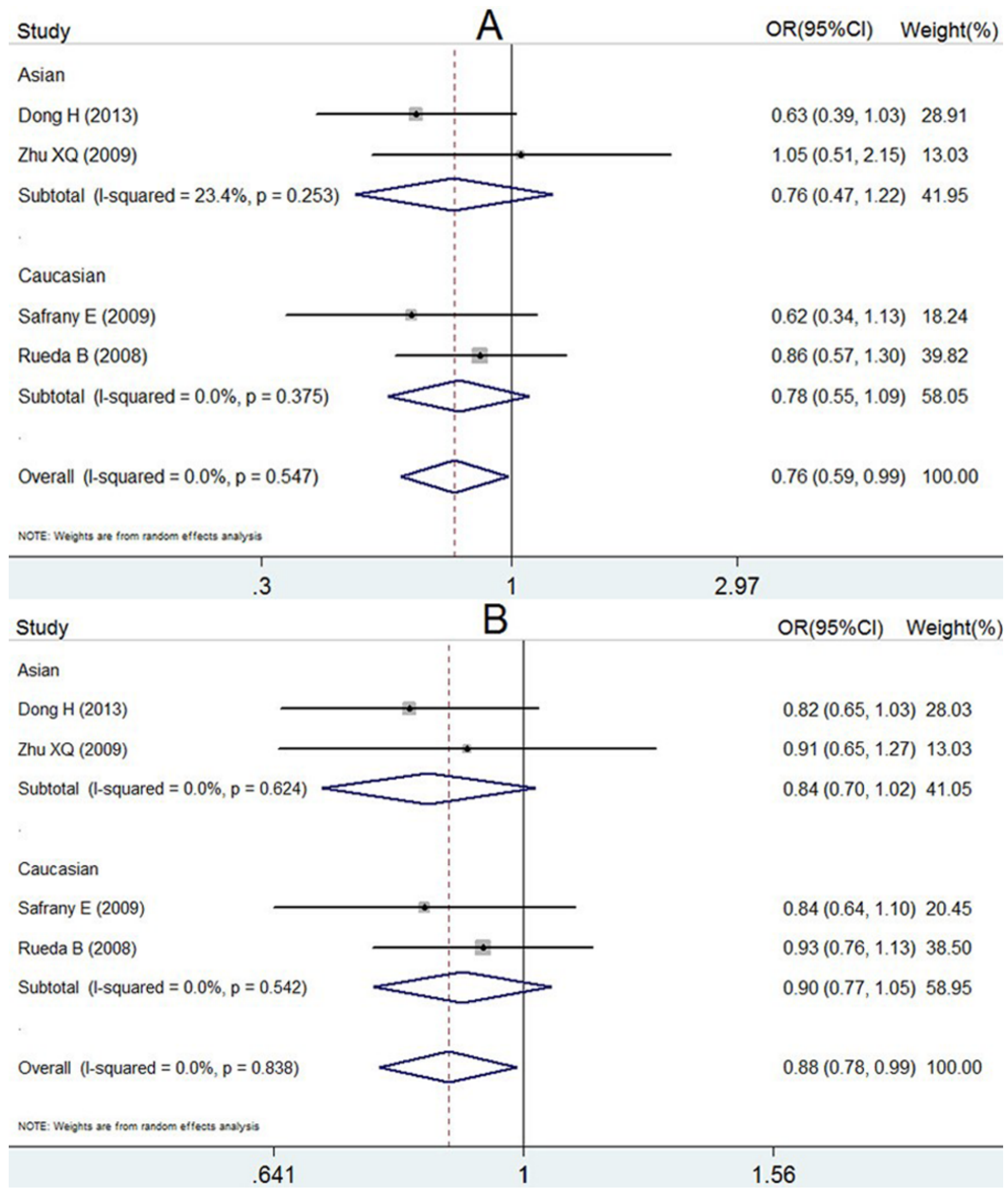




\section{3}

Forest plot of genetic association studies of rs2201841 homozygote model (CC vs. TT)

The horizontal axis: axis of Odds Ratio (OR);

The dotted line: mean value of overall OR

\begin{tabular}{|c|c|c|}
\hline Study & OR $(95 \% \mathrm{Cl})$ & Weight(\%) \\
\hline Zhu XQ (2009) & $0.69(0.25,1.88)$ & 34.76 \\
\hline Safrany E (2009) & $0.97(0.50,1.88)$ & 65.24 \\
\hline Overall (l-squared $=0.0 \%, p=0.578$ ) & $0.87(0.50,1.51)$ & 100.00 \\
\hline $\begin{array}{c}1 \\
.254\end{array}$ & $\begin{array}{c}1 \\
3.93\end{array}$ & \\
\hline
\end{tabular}




\section{Table 1 (on next page)}

The full search strategy for Embase 


\section{\#1 'interleukin'/exp OR interleukin AND 23}

\#2 il AND 23

\#3 \#1 OR \#2

\#4 ankylosing AND ('spondylitis'/exp OR spondylitis)

\#5 ankylopoietic AND ('spondylarthritis'/exp OR spondylarthritis)

\#6 ankylopoietic AND ('spondylitis'/exp OR spondylitis)

\#7 ankylosing AND ('spine'/exp OR spine)

\#8 ankylosing AND spondylitis

\#9 Ankylosing AND ('spondylarthritis'/exp OR spondylarthritis)

\#10 Ankylosing AND ('spondylarthrosis'/exp OR spondylarthrosis)

\#11 'ankylosis'/exp OR ankylosis AND ('spondylitis'/exp OR spondylitis)

\#12 Ankylotic AND ('spondylitis'/exp OR spondylitis)

\#13 Bechterew AND ('disease'/exp OR disease)

\#14 Bekhterev AND ('disease'/exp OR disease)

\#15 Morbus AND bechterew

\#16 Spinal AND ('ankylosis'/exp OR ankylosis)

\#17 'spine'/exp OR spine AND ('ankylosis'/exp OR ankylosis)

\#18 'spondylarthritis'/exp OR spondylarthritis AND ankylopoietica

\#19 'spondylarthritis'/exp OR spondylarthritis AND ankylosans

\#20 'spondylarthrosis'/exp OR spondylarthrosis AND ankylopoietica

\#21 'spondylitis'/exp OR spondylitis AND ankylopoetica

\#22 'spondylitis'/exp OR spondylitis AND ankylopoietica

\#23 Spondylitis, AND ankylosing

\#24 'spondyloarthritis'/exp OR spondyloarthritis AND ankylopoietica

\#25 Vertebral and ('ankylosis'/exp OR ankylosis)

\#26 \#4 OR \#5 OR \#6 OR \#7 OR \#8 OR \#9 OR \#10 OR \#11 OR \#12 OR \#13 OR \#14 OR \#15 OR \#16 OR \#17 OR \#18 OR \#19 OR \#20 OR \#21 OR \#22 OR \#23 OR \#24 OR \#25

\#27 'single nucleotide polymorphism'/exp

\#28 Polymorphism, AND single AND ('nucleotide'/exp OR nucleotide)

\#29 Single AND ('nucleotide'/exp OR nucleotide) AND polymorphism

\#30 \#27 OR \#28 OR \#29

\#31 \#3 AND \#26 AND \#30 
Table 2 (on next page)

Main characteristics of studies included in this meta-analysis

Author : first author; HWE: Hardy-Weinberg Equilibrium; 


\begin{tabular}{|cccccccccc|}
\hline author & year & country & ethnicity & SNP & $\begin{array}{c}\text { AS } \\
\text { Group }\end{array}$ & $\begin{array}{c}\text { Control } \\
\text { Group }\end{array}$ & $\begin{array}{c}\text { test } \\
\text { method }\end{array}$ & HWE & source \\
\hline Dong H & 2013 & China & Asian & rs7517847 & 291 & 312 & PCR-RFLP & Y & blood \\
Zhu XQ & 2009 & China & Asian & rs7517847 & 144 & 143 & PCR-HRM & Y & blood \\
Zhu XQ & 2009 & China & Asian & rs2201841 & 116 & 102 & PCR-HRM & Y & blood \\
Safrany E & 2009 & Hungary & Caucasian & rs7517847 & 206 & 235 & PCR-RFLP & Y & blood \\
& & & & rs2201841 & & & & & b \\
Rueda B & 2008 & Spanish & Caucasian & rs7517847 & 365 & 500 & Taqman & Y blood \\
\hline
\end{tabular}




\section{Table 3(on next page)}

Quality assessment of studies 


\begin{tabular}{|lcccc|}
\hline Study included & Selection & Comparability & Exposure & Total \\
\hline 1. Dong H (2013) $^{[17]}$ & 3 & 2 & 2 & 7 \\
2. Safrany E (2009) $^{[16]}$ & 3 & 2 & 2 & 7 \\
3. Zhu XQ (2009) $^{[19]}$ & 3 & 1 & 2 & 6 \\
4. Rueda B (2008) $^{[21]}$ & 3 & 1 & 2 & 6 \\
\hline
\end{tabular}


Table 4 (on next page)

Genotype distribution of rs7517847 and rs2201841 associated with IL-23

૫ : no available dataMAF: minor allelic frequencies 


\begin{tabular}{|c|c|c|c|c|c|c|c|c|c|c|}
\hline \multirow{2}{*}{ author } & \multirow{2}{*}{ ethnicity } & \multirow{2}{*}{ group } & \multicolumn{3}{|c|}{ rs7517847 } & \multicolumn{5}{|c|}{ rs2201841 } \\
\hline & & & TT & GT & GG & MAF & CC & CT & TT & MAF \\
\hline \multirow[t]{2}{*}{ Dong $\mathrm{H}$} & Asian & AS & 104 & 146 & 41 & 0.39 & - & - & - & - \\
\hline & & control & 98 & 153 & 61 & 0.44 & - & - & - & - \\
\hline \multirow[t]{2}{*}{ Safrany E } & Caucasian & AS & 67 & 115 & 24 & 0.40 & 26 & 89 & 91 & 0.34 \\
\hline & & control & 69 & 126 & 40 & 0.44 & 15 & 102 & 118 & 0.28 \\
\hline \multirow[t]{2}{*}{ Zhu XQ } & Asian & AS & 61 & 59 & 24 & 0.37 & 64 & 40 & 12 & 0.28 \\
\hline & & control & 48 & 77 & 18 & 0.40 & 54 & 41 & 7 & 0.27 \\
\hline \multirow[t]{2}{*}{ Rueda B } & Caucasian & AS & 140 & 172 & 53 & 0.38 & - & - & - & - \\
\hline & & control & 182 & 238 & 80 & 0.40 & - & - & - & - \\
\hline
\end{tabular}




\section{Table 5 (on next page)}

Meta-analysis of polymorphisms of rs 7517847

n: number of studies included; 


\begin{tabular}{|c|c|c|c|c|c|}
\hline SNP & $\mathbf{n}$ & Genetic model & OR (95\% CI) & $\mathbf{P}$ & $\mathbf{I}^{2}$ \\
\hline \multirow[t]{5}{*}{ rs7517847 } & 4 & $\begin{array}{c}\text { homozygote model } \\
\text { GG vs TT }\end{array}$ & $0.76(0.59,0.99)$ & 0.038 & $0 \%$ \\
\hline & & $\begin{array}{c}\text { heterozygote model } \\
\text { GT vs TT }\end{array}$ & $0.87(0.73,1.05)$ & 0.157 & $0 \%$ \\
\hline & & $\begin{array}{l}\text { allelic model } \\
\text { G vs T }\end{array}$ & $0.88(0.78,0.99)$ & 0.032 & $0 \%$ \\
\hline & & $\begin{array}{l}\text { recessive model } \\
\text { GG vs }(\mathrm{TT}+\mathrm{GT})\end{array}$ & $0.82(0.65,1.03)$ & 0.090 & $28.2 \%$ \\
\hline & & $\begin{array}{l}\text { dominant model } \\
(\mathrm{GG}+\mathrm{GT}) \text { vs TT }\end{array}$ & $0.85(0.71,1.01)$ & 0.066 & $0 \%$ \\
\hline
\end{tabular}




\section{Table 6 (on next page)}

Meta-analysis of polymorphisms of rs2201841

n: number of studies included; 
2

\begin{tabular}{|c|c|c|c|c|c|}
\hline SNP & $\mathbf{n}$ & Genetic model & OR (95\% CI) & $\mathbf{P}$ & $\mathbf{I}^{2}$ \\
\hline \multirow[t]{5}{*}{ rs2201841 } & 2 & $\begin{array}{c}\text { homozygote model } \\
\text { CC vs. TT }\end{array}$ & $0.87(0.50,1.51)$ & 0.631 & $0 \%$ \\
\hline & & $\begin{array}{c}\text { heterozygote } \\
\text { model } \\
\text { CT vs TT }\end{array}$ & $0.93(0.62,1.40)$ & 0.726 & $4.70 \%$ \\
\hline & & $\begin{array}{l}\text { allelic model } \\
\text { C vs T }\end{array}$ & $0.99(0.77,1.27)$ & 0.915 & $0 \%$ \\
\hline & & $\begin{array}{l}\text { recessive model } \\
\text { CC vs (TT+CT) }\end{array}$ & $1.04(0.69,1.55)$ & 0.866 & $0 \%$ \\
\hline & & $\begin{array}{l}\text { dominant model } \\
(\mathrm{CC}+\mathrm{CT}) \text { vs TT }\end{array}$ & $0.94(0.64,1.38)$ & 0.746 & $0 \%$ \\
\hline
\end{tabular}

3 


\section{Table 7 (on next page)}

Results of subgroup analysis of rs 7517847 by ethnicity 


\begin{tabular}{|c|c|c|c|c|c|}
\hline \multirow{3}{*}{$\begin{array}{l}\text { SNP } \\
\text { rs7517847 }\end{array}$} & \multirow{3}{*}{ Genetic model } & \multicolumn{4}{|c|}{ Ethnicity } \\
\hline & & \multicolumn{2}{|c|}{ Asian } & \multicolumn{2}{|c|}{ Caucasian } \\
\hline & & OR $(95 \% \mathrm{CI})$ & $P$ value & OR $(95 \% \mathrm{CI})$ & $P$ value \\
\hline & $\begin{array}{l}\text { homozygote model } \\
\text { (GG/ TT) }\end{array}$ & $0.74(0.50-1.10)$ & 0.140 & $0.78(0.55-1.09)$ & 0.143 \\
\hline & $\begin{array}{l}\text { heterozygote model } \\
\text { (GT/ TT) }\end{array}$ & $0.79(0.59-1.05)$ & 0.109 & $0.94(0.74-1.20)$ & 0.613 \\
\hline & $\begin{array}{l}\text { recessive model } \\
(\mathrm{GG} / \mathrm{GT}+\mathrm{TT})\end{array}$ & $0.84(0.59-1.20)$ & 0.335 & $0.80(0.59-1.09)$ & 0.161 \\
\hline & $\begin{array}{l}\text { dominant model } \\
(\mathrm{GG}+\mathrm{GT} / \mathrm{TT})\end{array}$ & $0.78(0.59-1.02)$ & 0.071 & $0.90(0.72-1.13)$ & 0.373 \\
\hline & $\begin{array}{l}\text { allelic model } \\
(\mathrm{G} / \mathrm{T})\end{array}$ & $0.84(0.70-1.02)$ & 0.080 & $0.90(0.77-1.05)$ & 0.181 \\
\hline
\end{tabular}

2

3 